\title{
THE EFFECT OF GLYCOSIDASES ON THE SURVIVAL OF RAT ERYTHROCYTES IN CIRCULATION*,+
}

Karol A. Gutowski, Daniel A. Linseman, And David AminofF ${ }^{\ddagger}$

Institute of Gerontology and Department of Biological Chemistry, The University of Michigan, Ann Arbor, Michigan 48109 (U.S.A.)

(Received December 15th, 1986; accepted for publication in revised form, June 29th, 1987)

\section{ABSTRACT}

Enzymic removal of sialyl groups from mammalian erythrocytes resulted in their rapid sequestration from circulation subsequent to autologous transfusion. It has been demonstrated by many investigators that the terminal $\beta$-D-galactosyl group, exposed on red blood cell by in vitro desialosylation, is recognized by an autoimmune anti-galactosyl IgG and/or by a lectin-like receptor on monocytes and macrophages. It is demonstrated herein that the disaccharide structure $\beta-\mathrm{D}-\mathrm{Gal} p$ $(1 \rightarrow 3)-D-G a l p N A c(a)$ is masked in normal rat RBC, but exposed in asialo-RBC; (b) could be detected with fluorescently-labeled peanut agglutinin; (c) could be releascd from the asialo-RBC with an endo- $N$-acctyl- $\alpha$-D-galactosaminidasc; and (d) upon its removal by treatment with the endo- $N$-acetyl- $\alpha$-D-galactosaminidase, enhances the survival of the asialo- $\mathrm{RBC}$ in circulation.

\section{INTRODUCTION}

Enzymic removal of sialyl groups from mammalian erythrocytes (RBC) resulted in their rapid sequestration from circulation ${ }^{1}$. With the demonstration that the sialic acid content of RBCs decreases with their in vivo aging in circulation ${ }^{2}$, it has been postulated that a removal of sialyl groups might be involved in in vivo aging of RBC, with consequent sequestration of the RBC when the sialic acid content dropped by $10-15 \%$ to a threshold value ${ }^{3}$. Evidence from a number of studies in our laboratory has shown that in both artificial removal of sialyl groups from $\mathrm{RBC}$ with sialidase from Vibrio cholerae, $\mathrm{aRBC}$, and in vivo aging of $\mathrm{RBC}$ in circulation, the loss of sialic acid groups is accompanied by the unmasking of the penultimate $\beta$-D-galactosyl residues. This is analogous to the Ashwell and Morell system for the sequestration of asialo plasma glycoproteins by hepatocytes ${ }^{4}$. In

\footnotetext{
${ }^{*}$ Dedicated to Professor Walter T. J. Morgan.

'This study was supported by grant HL-AM-17881 from the National Institutes of Health, the Juvenile Diabetes Foundation, and a Biomedical Research Support Grant from the University of Michigan Medical School.

†To whom correspondence should be addessed.
} 
contrast, we have shown that asialo RBC involve interaction with liver Kupffer cells and spleen monocytes ${ }^{5}$.

We believe that the in vivo aging of $\mathrm{RBC}$ in circulation involves the desialosylation of glycophorin $\mathrm{A}$, the most abundant sialoglyconjugate on human $\mathrm{RBC}$ surface. The hypothesis is supported by the isolation ${ }^{6}$ of a "senescence factor glycopeptide" (SFG) from the tryptic digest of intact human RBC. This glycopeptide, free of all sialic acid groups, had all the properties expected of the "senescence factor" with the capacity to inhibit erythrophagocytosis of senescent RBCs by autologous human blood monocytes in nanomolar concentrations, in contrast to D-galactose alone, which inhibited at millimolar concentrations, and $\beta$-D-galactosyl groups bound to bovine serum albumin which inhibited at micromolar concentrations?.

The well known susceptibility of glycophorin A to tryptic digestion ${ }^{8,9}$, and the method of purification devised ${ }^{6}$ for the isolation of SFG would point to glycophorin $A$ as the putative parent molecule of SFG on human and other mammalian RBC surfaces. Nonetheless, it could be argued that SFG still was contaminated with a potent inhibitor of erythrophagocytosis of an as yet unidentified origin. The objective of this communication is to provide yet another and more direct approach to elucidate the role of glycophorin-like structures, recently characterized ${ }^{10}$ in rat $\mathrm{RBC}$, in the sequestration of asialo RBC. Here, we focus on the disaccharide structure, $\beta$-D-Galp-( $\rightarrow 3)$-D-Gal $p$ NAc, and demonstrate that: (a) It is masked in normal RBC, but exposed in asialo RBC (aRBC); (b) it can be detected with fluorescein isothiocyanate-labeled peanut agglutinin (FITC-PNA); (c) it can be released from the aRBC with endo- $N$-acetyl- $\alpha$-D-galactosaminidase (endoglycosidase) $)^{11,12}$; and (d) upon its release with the endoglycosidase treatment, the survival of the aRBC in circulation is enhanced ${ }^{13,14}$. As previously, the SpragueDawley rat was used as the experimental model.

EXPERIMENTAL

Materials. - The following enzymes were obtained from the sources indicated: $\beta$-D-galactosidase from bovine testes was a gift from Dr. G. W. Jourdian, University of Michigan ${ }^{15} ; V$. cholerae sialidase was obtained from Worthington Biochemical Corp.; and endo- $N$-acetyl- $\alpha$-D-galactosaminidase was prepared in our laboratory from Clostridium perfringens ${ }^{11.12}$. FITC-PNA, was obtained from Sigma Chemical Co. (St. Louis, MO); and Sprague-Dawley rats from Charles River Breeding Laboratories, Inc. (Wilmington, MA). $\mathrm{Na}_{2}{ }^{51} \mathrm{CrO}_{4}$ used for in vitro labeling of RBC was purchased from E. R. Squibb and Sons, Inc. (Princeton, NJ). Treatment of rat $R B C$ with enzymes to determine their effect on the survival of $R B C$ in circulation

Incubation of rat RBC with glycosidases. - Whole blood was obtained in a heparinized syringe by cardiac puncture of an approximately $400 \mathrm{~g}$, male SpragueDawley rat. The blood was centrifuged, and the plasma and buffy coat were dis- 
carded. The remaining packed cells were washed three times with isotonic saline solution. Four equivolume samples of RBC were incubated with the following reagents made up in isotonic saline solution at $\mathrm{pH}$ 5.5: (a) Isotonic saline control, (b) sialidase from $V$. cholerae ( 0.2 units $/ \mathrm{mL}$ of packed cells), (c) sialidase from $V$. cholerae $(0.2$ units $/ \mathrm{mL}$ of packed cells) and endoglycosidase $(0.15$ units $/ \mathrm{mL}$ of packed cells), and (d) sialidase from $V$. cholerae $(0.2$ units $/ \mathrm{mL}$ of packed cells) and $\beta$-D-galactosidase from bovine testes $(0.15$ units $/ \mathrm{mL}$ of packed cells $)$. The incubations were carried out for $1 \mathrm{~h}$ at $37^{\circ}$.

Quantitative determination of FITC-PNA adsorption on enzymically treated rat $R B C$. - Upon completion of the incubation period, the samples were centrifuged and the supernatant discarded. The samples then were washed twice with phosphate-buffered saline solution (pH 7.3) (PBS). Aliquots of packed cells containing $1 \times 10^{7}$ erythrocytes were removed from each of the samples and diluted to a volume of $100 \mu \mathrm{L}$ with PBS. To these dilute red cell suspensions was added a $1: 20$ dilution of FITC-PNA of $0.1 \%$ solution in PBS $(100 \mu \mathrm{L})$. The resulting $200-\mu \mathrm{L}$ samples in triplicate were incubated for $1 \mathrm{~h}$ at $4^{\circ}$, and then centrifuged. Equal volumes of supernatant were removed from each sample and diluted to a total volume of $3 \mathrm{~mL}$ with isotonic saline solution adjusted to $\mathrm{pH} 9.0$ to enhance the fluorescence. The fluorescence was measured with a Turner Model 430 spectrofluorometer at excitation and emission settings of $498 \mathrm{~nm}$ and $534 \mathrm{~nm}$, respectively. The measured fluorescence is that of unadsorbed fluorescent lectin. The determination was made possible by comparison to a set of calibration standards of the $1: 20$ dilution of $0.1 \%$ FITC-PNA in isotonic saline solution adjusted to $\mathrm{pH} 9.0$. The amount of adsorbed FITC-PNA then was calculated by subtraction of the amount not adsorbed from the total FITC-PNA added to the sample. This procedure was adopted in order to shorten the assay. Previous experiments indicated that all the adsorbed FITC-PNA could be quantitatively eluted with $0.4 \mathrm{~m}$ laclose. Results are shown in Table I.

Survival of radioactively-labeled and enzymically treated rat $R B C$ in circulation. - Comparable enzymically-treated RBCs (Samples a-d), 4.0-6.5 $\times 10^{9} \mathrm{RBC}$, were washed and incubated for $0.5 \mathrm{~h}$ at $37^{\circ}$ with $\mathrm{Na}_{2}{ }^{51} \mathrm{CrO}_{4}(50 \mu \mathrm{Ci})$ to label the RBC. The resulting incubation mixtures were centrifuged to remove the unincorporated $\mathrm{Na}_{2}{ }^{51} \mathrm{CrO}_{4}$ and washed until no more perceptible radioactivity remained in the washes; $\sim 95 \%$ of the radioactivity was incorporated into the RBC. Each pellet of RBCs was suspended in saline solution $(\sim 1 \mathrm{~mL})$ and the $R B C$ suspension $(2 \mathrm{~mL})$ injected intraperitoneally into each rat $^{13,14}$. Enough $\mathrm{RBC}$ incubation mixtures of a-d were prepared to inject two rats per treatment. After $24 \mathrm{~h}$, blood $(20 \mu \mathrm{L})$ was removed from the tail of each rat and suspended in saline solution (480 $\mu \mathrm{L}$ ). Similar blood samples were removed at daily intervals, and all samples were stored at $4^{\circ}$. The radioactivity was counted simultaneously at the end of the experiment in a Packard gamma-counter. The per cent of radioactivity remaining in the blood samples was computed as a percentage of that found at the end of the first $24 \mathrm{~h}$. The results are shown in Fig. 1. 
TABLE I

FITC PNA ADSORPTION" AS A PERCENTAGE OF THAT OBSERVED FOR ASIAI.O RAT ERYTHROCYTES

\begin{tabular}{llccc}
\hline Sample & $\begin{array}{l}\text { Isotonic } \\
\text { saline solution }\end{array}$ & Sialidase & $\begin{array}{l}\text { Sialidase plus } \\
\text { endoglycosidase }\end{array}$ & $\begin{array}{l}\text { Sialidase plus } \\
\text { B-1)-galactosidase }\end{array}$ \\
\hline $\mathrm{n}$ & 3 & 3 & 3 & 3 \\
$\mathrm{x}(\%)$ & 0 & 100 & 12.4 & 55.0 \\
$\sigma_{\mathrm{x}}$ & & & 10.2 & 35.3
\end{tabular}

${ }^{a}$ Determined by subtraction. see Experimental section.

RESULTS

In view of the marked difference in the molecular extinction coefficient of sialic acid in the TBA assay ${ }^{16}$, the disaccharide in the modified Morgan-EIson assay $^{17}$, and D-galactose with the galactose-dehydrogenase assay ${ }^{18}$, it was impractical to photometrically monitor the action, on the aRBC of the endo-glycosidase and bovine testes $\beta$-D-galactosidase. Instead, the process was monitored by determining the amount of FITC-PNA that was adsorbed on the $\mathrm{ARBC}^{19}$. The peanut agglutinin was chosen deliberately to monitor the changes on $\mathrm{RBC}$ surface resulting from the enzymic treatments. This lectin focuses our attention only on the D-galactosyl group (of the many exposed by removal of sialyl groups from RBC) that is present as the disaccharide residue, $\beta-\mathrm{D}-\mathrm{Gal} p-(1 \rightarrow 3)$-D-GalpNAc $\mathrm{N}^{20}$. Normal rat RBCs do not adsorb significant amounts of FITC-PNA; after desialosylation,

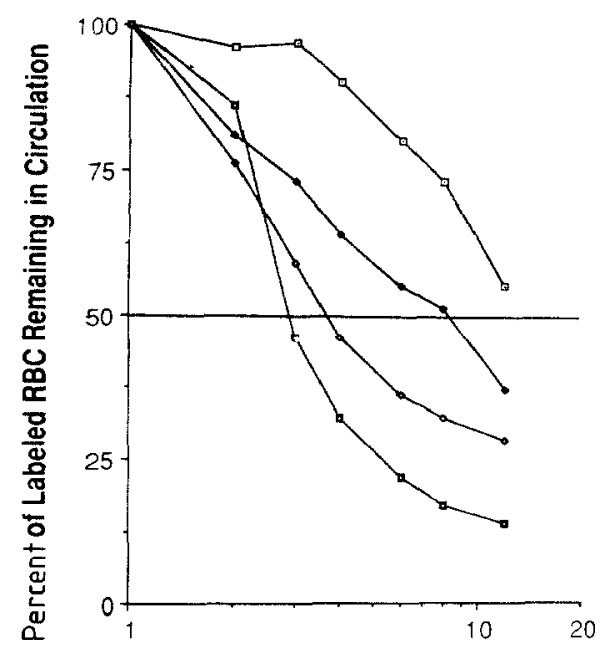

Days Post i.p. Injection of Labeled RBC

Fig. 1. Survival of enzymically treated rat $R B C$ in circulation: $(-\square-\square-\square)$ Control RBC, in isotonic RBC at pH 5.5; (- $\square-\square-\square-)$ sialidase-treated RBC; $(-)$ sialidase- plus endoglycosidase-treated RBC: and $\left.(-\diamond-\diamond-\rangle_{-}\right)$sialidase- plus $\beta$-D-galactosidase-treated RBC. 
they are very reactive, and the amount of lectin adsorbed is proportional to the number of aRBCs present. Under our standardized conditions, we arbitrarily assigned the amount of FITC-PNA bound to RBC as $100 \%$, and then determined the effect of the two enzymes, $\beta$-D-galactosidase from bovine testes and the $\mathrm{Cl}$. perfringens endoglycosidase, on the extent of FITC-PNA that still could bind, giving us a figure of 55 and $12 \%$, respectively (Table I). Within the limitations of the data presented and under the conditions of this assay at $\mathrm{pH} 5.5$ and $37^{\circ}$, it is apparent that the endoglycosidase is more effective than the $\beta$-D-galactosidase in removing the PNA reactive sites.

We have demonstrated previously ${ }^{13,14}$ that intraperitoneal transfusion is an extremely useful procedure for the transfer of relatively large numbers of RBCs. Normal untreated RBCs are recovered almost quantitatively in the general circulation within $22 \mathrm{~h}$, whereas aRBCs appear preponderantly in the liver with only a few cells remaining in the peritoneal cavity. By use of the same procedure, it was possible to monitor the survival of the enzyme-treated and -untreated RBCs remaining in circulation over a 12-day period. The mean results from two experiments are shown in Fig. 1, from which one may readily compare (a) the $50 \%$ survival of RBCs in circulation, and (b) the per cent of RBCs surviving after 12 days, namely, $55 \pm 10 \%$ for the untreated control RBCs, $12 \pm 4 \%$ for the sialidase-treated RBCs, $37 \pm 10 \%$ for the sialidase- plus endoglycosidase-, and $28 \pm 9 \%$ for the sialidaseplus $\beta$-D-galactosidase-treated RBCs, respectively.

DISCUSSION

The mechanism whereby RBCs are removed at the end of their life-span in circulation has been an intriguing question for many years. Briefly, the evidence favoring a role for the sialoglycoconjugates in RBC sencscence and sequestration comes from the following six lines of investigation ${ }^{21}$ : (a) Analysis of the carbohydrate composition of young and old RBCs, (b) use of asialo-RBC as a model for $\mathrm{RBC}$-senescence, (c) identification of the interaction of immunoglobulin with senescent RBCs and aRBCs, (d) studies on the inhibition of interaction of senescent RBCs with macrophages and immunoglobulins, (e) correlations derived from a study of the RBC sialoglycoconjugates in the anemias, and (f) isolation of a putative senescence factor glycopeptide.

The circumstantial evidence thus collected implicates the involvement of a change in the chemical structure of the RBC membrane, as well as a change in its physical properties. A number of investigators have come to the same conclusion, but ascribe ${ }^{22}$ the nature of the chemical change either to the involvement of Band 3 , the exposure of a terminal $\alpha$-D-galactosyl group ${ }^{23}$, or to the $\beta$-D-Gal $p-(1 \rightarrow 3)$-DGalpNAc group of a glycophorin-like molecule ${ }^{21}$. Our evidence in support of a glycophorin-like molecule can be summarized as follows: (a) The presence of more galactose oxidase and sialyltransferase receptor sites in senescent as compared to young RBCs (ref. 3); (b) the ability to isolate ${ }^{6}$ from senescent, but not young. 
RBCs a "senescence-factor glycopeptide" (SFG); (c) indications ${ }^{6}$ from those properties of SFG utilized for its isolation as well as its reactivity with spleen monocytes, that SFG is biologically active; (d) the destruction of SFG's interaction with spleen monocytes after its treatment with $\beta$-D-galactosidase, attesting to the significant role of galactosyl residues in this biological activity ${ }^{6}$; (e) the ability of SFG to inhibit erythrophagocytosis at nanomolar concentrations, as compared to D-galactose at millimolar and D-galactosyl groups bound to BSA at micromolar concentrations $^{7}$; and (f) the ability of injected SFG from human senescent RBC to inhibit the uptake of mouse senescent RBC at the end of the RBC life-span ${ }^{24}$.

The aforementioned data are compatible with the hypothesis that a glycophorin-like molecule, devoid of sialic acid groups, could be the putative recognition factor on senescent RBCs. Since the homogeneity of SFG has not as yet been established $^{6}$, it could be argued that it is not necessarily a component of the glycophorin molecule that is involved, but another highly potent "senescence factor", which would still contaminate the glycophorin glycopeptides that were isolated from the RBC-tryptic digest ${ }^{6}$ and used for the inhibition studies? ${ }^{7}$. We have succeeded in showing, however, that the reduced survivability of aRBCs can be restored by removing the disaccharide groups. This "rejuvenation" of aRBCs by treatment with the endoglycosidase indicates that only the disaccharide groups exposed by the desialosylation of RBCs are detected by the reticulo-endothelial system.

Work in our laboratories and those of others has demonstrated significant differences in the behavior of asialo and senescent $\mathrm{RBCs}^{21}$. We attributed some of these differences primarily to the amount of sialic acid removed from the RBC. The observation that we can reverse the sequestration of aRBC by treatment with the endoglycosidase now provides us with a valuable tool to further explore this problem and eludicate the mechanism whereby senescent RBCs are recognized and sequestered.

\section{REFERENCES}

1 D. Aminoff, W. C. Bell, I. Fulton, and N. Ingebrigtsen, Am. J. Hematol, 1 (1976) 419-432.

2 D. DANON. Bibl. Haematol. (Basel), 29 (1968) 178-188.

3 D. aminoff, M. A. Ghalambor, and C. J. Henrich, in W. C. Kruckberg, J. W. Eaton. and G. J. BREWER (Eds.), Erythrocyte Membranes, Recent Clinical and Experimental Advances, Vol. 2. Liss, Ncw York, 1981, pp. 269-278.

4 G. Ashwell and A. G. Morell, Adv. Enzymol., 41 (1974) 99-128.

5 D. Aminofy, W. F. Vorder Bruegge, W. C. Bell, K. Sarpolis, and R. Williams, Proc. Natl. Acad. Sci. U.S.A., 74 (1977) 1521-1524.

6 C. J. Henrich and D. Aminoff, Carbohydr. Res., 120 (1983) 55-66.

7 J. Vaysse, L. Gattegno, D. Bladier. and D. Aminoff, Proc. Natl. Acad. Sci. U.S.A., 83 (1986) $1339-1343$.

8 D. B. THOMAS AND R. J. Winzler, J. Biol. Chem., 244 (1969) $5943-5946$.

9 M. Tomita and V. T. Marchesi, Proc. Natl. Acad. Sci. U.S.A., 72 (1975) 2964-2968.

10 A. S. B. Edge, A. VAn Langenhove, V. Reinhold. And P. Weber, Biochemistry, 25 (1986) 80178024.

11 C. C. Huang and D. Aminoff, J. Biol. Chem., 247 (1972) 6737-6742. 
12 W. C. Bell, N. Pomato, And D. Aminoff, Carbohydr. Res., 61 (1978) 447-455.

13 B. Smedsrod and D. Aminoff, Am. J. Hematol., 15 (1983) 123-133.

14 B. Smedsrod and D. Aminoff, Am. J. Hematol., 18 (1985) 31-40.

15 J. J. Distler and G. W. Jourdian, J. Biol. Chem., 248 (1973) 6772-6780.

16 D. AMinurt, Biochem. J., 81 (1961) 384-392.

17 P. M. T. HANSEN, J. Dairy Sci., 50 (1967) 952.

18 P. R. Finch, R. Yuen, H. Schachter, and M. A. Moscarello, Anal. Biochem., 31 (1969) 296305.

19 M. Monsigny, C. Sene, And A. Obrenovitch, Eur. J. Biochem., 96 (1979) 295-300.

20 I. J. Goldstein and C. E. Hayes, Adv. Carbohydr. Chem. Biochem., 35 (1978) 127-340.

21 D. Aminoff, in J. W. Eaton, D. K. Konzen, And J. G. White (Eds.), Cellular and Molecular Aspects of Aging; The Red Cell as a Model, Alan R. Liss, New York, 1985, pp. 279-300.

22 M. M. B. KAY, in J. W. EATON, D. K. KONZEN, AND J. G. White (Eds.), Cellular and Molecular Aspects of Aging: The Red Cell as a Model, Alan R. Liss, New York, 1985, pp. 251-262.

23 U. Galili, I. Fletchner, and E. A. Rachmilewitz, in J. W. Eaton, D. K. Konzen, and J. G. Whtte (Eds.), Cellular and Molecular Aspects of Aging: The Red Cell as a Model, Alan R. Liss, New York, 1985, pp. 263-276.

24 D. Bladier, J. Vaysse, R. Vassy, L. Gattegno, and D. Aminoff, unpublished results. 